\title{
Tricritical Phenomena in a Z(3) Lattice Gauge Theory
}

\author{
N.S. Ananikian* \\ Department of Theoretical Physics, Yerevan Physics Institute, \\ Alikhanian Br. 2, 375036 Yerevan, Armenia \\ and \\ R.R. Shcherbakov ${ }^{\dagger \ddagger}$ \\ Bogoliubov Laboratory of Theoretical Physics, \\ JINR, 141980 Dubna, Russia
}

\begin{abstract}
The Z(3) gauge model with double plaquette representation of the action on a generalized Bethe lattice of plaquettes is constructed. It is reduced to the spin-1 Blume-Emery-Griffiths (BEG) model. An Ising-type critical line of a second-order phase transition ending in the tricritical point is found.
\end{abstract}

*E-mail address: nanan@tpd.erphy.armenia.su

†E-mail address: shcher@thsun1.jinr.dubna.su

${ }^{\ddagger}$ On leave of absence from Department of Theoretical Physics, Yerevan Physics Institute, Armenia 
A continuum limit of a lattice gauge theory may be constructed in the points of a second-order phase transition, since infinite range correlations allow us to wipe out lattice effects.

At a tricritical or multicritical points the number of relevant couplings is larger than at the vicinity of the second-order critical line. Therefore the existence of such points in the lattice gauge theories opens a possibility for further non-trivial continuum limits.

The purpose of this paper is a search for multicritical points in the strongcoupling region of the $\mathrm{Z}(3)$ lattice gauge theory.

According to Kogut's formulation of a gauge Potts model on a lattice [1], we constructed the Z(3) gauge model on the generalized Bethe lattice of plaquettes [2, 3] with double plaquette (window) representation of the action. The choice of this mixed action allowed us to connect it with the Hamiltonian of the spin-1 BEG model [4] and to receive the $\lambda$-line of the second-order phase transition ending in the tricritical point.

This lattice is a generalization of the Cayley tree. For our purpose we consider it only with coordination number (the number of plaquettes coming out from one link) 2. The dual lattice is constructed by joining each nearest centers of plaquettes and as a result we get the usual Bethe lattice with coordination number 4 .

Dual lattices for the generalized Bethe ones with coordination numbers higher than 2 are more complicated hierarchical lattices. For example, if we consider the generalized Bethe lattice with coordination number 3 then the dual lattice will be a Husimi lattice [5].

The enlarged lattice gauge actions involving new double plaquette interaction terms were proposed and studied in $3 d$ and $4 d$ by Edgar [6], Bhanot et al [7]. The $2 d$ version of one of these lattice gauge models with $\mathrm{Z}(2)$ gauge symmetry formulated on the planar rectangular windows was investigated by Turban [8]. This model with pure gauge action had been reduced to the usual spin- $\frac{1}{2}$ Ising model on the square lattice and the point of a second-order phase transition was found.

The model is considered on the generalized Bethe lattice in terms of the bond variables $U_{b}$ which take their values in the $\mathrm{Z}(3)$, the group of the third roots of unity. Let $U_{p_{i}}=\prod_{b \in \partial p} U_{b}$ denote the product of $U_{b}$ 's around an elementary plaquette $i$.

The gauge action of the model is

$$
S_{\text {Gauge }}\left(\beta_{2 g}, \beta_{2 g}^{\prime}, \beta_{g}\right)=S_{p p}+S_{p},
$$

where

$$
\begin{gathered}
S_{p p}=-\sum_{<p_{i} p_{j}>}\left\{\beta_{2 g}\left(\delta_{U_{p_{i}}, 1} \delta_{U_{p_{j}}, 1}+\delta_{U_{p_{i}}, z} \delta_{U_{p_{j}}, z}\right)+\beta_{2 g}^{\prime}\left(\delta_{U_{p_{i}}, 1} \delta_{U_{p_{j}}, z}+\delta_{U_{p_{i}}, z} \delta_{U_{p_{j}}, 1}\right)\right\} \\
S_{p}=\beta_{g} \sum_{p_{i}}\left(\delta_{U_{p_{i}}, 1}+\delta_{U_{p_{i}}, z}\right)
\end{gathered}
$$

$U_{p_{i}}$ denotes the usual plaquette variable, the product of link gauge fields $U_{x, \mu}$ around an elementary plaquette. The first summation goes over all nearest-neighbor plaquettes and the second one is over all plaquettes of the lattice, $z=\exp \left(i \frac{2 \pi}{3}\right) \in Z(3)$. 
Introducing spin variables $S_{i}$ in the sites of the dual lattice such that

$$
\begin{aligned}
& S_{i}=\delta_{U_{p_{i}}, 1}-\delta_{U_{p_{i}}, z}, \\
& S_{i}^{2}=\delta_{U_{p_{i}}, 1}+\delta_{U_{p_{i}}, z}
\end{aligned}
$$

the action (11) becomes

$$
S_{\text {Spin }}\left(\beta_{2 g}, \beta_{2 g}^{\prime}, \beta_{g}\right)=-\sum_{<i j>}\left\{\frac{\beta_{2 g}-\beta_{2 g}^{\prime}}{2} S_{i} S_{j}+\frac{\beta_{2 g}+\beta_{2 g}^{\prime}}{2} S_{i}^{2} S_{j}^{2}\right\}+\beta_{g} \sum_{i} S_{i}^{2}
$$

in which we recognize the Hamiltonian multiplied by $1 / k_{B} T$ of the well known BEG model 4 .

The corresponding partition function of the model (1) on the generalized Bethe lattice is

$$
Z_{\text {Gauge }}\left(\beta_{2 g}, \beta_{2 g}^{\prime}, \beta_{g}\right)=\sum_{\{U\}} \exp \left[-S_{\text {Gauge }}\left(\beta_{2 g}, \beta_{2 g}^{\prime}, \beta_{g}\right)\right]
$$

where the sum is taken over all possible configurations of the gauge variables $\{U\}$. This partition function can be rewritten in terms of the spin variables $S_{i}$ defined in the sites of the dual lattice

$$
Z_{\text {Gauge }}=3^{N} Z_{\text {Spin }}^{\text {Dual }}
$$

where

$$
Z_{\text {Spin }}^{\text {Dual }}=\sum_{\{S\}} \exp \left[-S_{S p i n}\right]
$$

A factor $3^{N}$ has been included in the equation (5) to take into account the difference between the number of gauge $\{U\}$ and spin $\{S\}$ configurations, since for each spin configuration with $\mathrm{N}$ sites we have $3^{N}$ identical gauge ones.

A gauge invariant quantity $<\delta_{U_{p_{i}, 1}}+\delta_{U_{p_{i}, z}}>$ is the order parameter of the model.

The BEG model on the usual Bethe lattice have been exactly solved in [9, 10], where the $\lambda$-line of the second-order phase transition ending in the tricritical point have been found. Thus we can rewrite these solutions in terms of the gauge couplings. For the $\lambda$-line we have

$$
\begin{gathered}
\exp \left(\beta_{g}^{\lambda}\right)=2\left(b-u_{0}\right)\left(u_{0}+1\right)^{3} u_{0}^{-1} \\
<\delta_{U_{p_{i}, 1}}+\delta_{U_{p_{i}, z}}>_{\lambda}=\frac{u_{0}\left(1+u_{0}\right)}{b+u_{0}^{2}}
\end{gathered}
$$

where

$$
\begin{gathered}
b=\exp \left(\frac{\beta_{2 g}+\beta_{2 g}^{\prime}}{2}\right) \cosh \left(\frac{\beta_{2 g}-\beta_{2 g}^{\prime}}{2}\right)-1, \\
a=b /\left(\exp \left(\frac{\beta_{2 g}+\beta_{2 g}^{\prime}}{2}\right) \sinh \left(\frac{\beta_{2 g}-\beta_{2 g}^{\prime}}{2}\right)\right), \\
u_{0}=a /(3-a)
\end{gathered}
$$


The tricritical point is determined by the following expression

$$
\frac{u_{0}+1}{b-u_{0}}=2+\frac{1}{8 u_{0}} .
$$

Fig.1 shows the phase diagram of the $\mathrm{Z}(3)$ gauge model for the value $\beta_{2 g}^{\prime} / \beta_{2 g}=\frac{1}{2}$. The $\lambda$-line (solid line) of the second-order phase transition starts at $\left(\beta_{g}=-\infty, \beta_{2 g}=\right.$ $2 \log 2$ ), and finishes at the tricritical point $\mathrm{A}$ which exists for the cases $-\infty<$ $\beta_{2 g}^{\prime} / \beta_{2 g}<0.5478$. The tricritical point A cuts the $\lambda$-line and separates the secondorder phase transition from the first-order one.

At the end we want to write down the tricritical exponents:

$$
\begin{aligned}
& <\delta_{U_{p_{i}, 1}}+\delta_{U_{p_{i}, z}}>-<\delta_{U_{p_{i}, 1}}+\delta_{U_{p_{i}, z}}>_{\lambda} \sim\left|\beta_{g}-\beta_{g}^{T r i}\right|^{\frac{1}{2}}, \\
& <\delta_{U_{p_{i}, 1}}+\delta_{U_{p_{i}, z}}>-<\delta_{U_{p_{i}, 1}}+\delta_{U_{p_{i}, z}}>_{\lambda} \sim\left|\beta_{2 g}-\beta_{2 g}^{T r i}\right| .
\end{aligned}
$$

We constructed the $\mathrm{Z}(3)$ gauge lattice model with double plaquette (window) representation of the action and showed that this model is dual to the spin-1 BEG

one. Using the exact solution of the BEG model on the Bethe lattice we found the line of the second-order phase transition ending in the tricritical point.

We would like to thank R. Flume, K. Oganessyan and A. Akheyan for fruitful discussions.

One of us (R.R.S.) wishes to thank V.B. Priezzhev for the hospitality extended to him at the Bogoliubov Laboratory of Theoretical Physics of Joint Institute for Nuclear Research where this work has been done.

This work was partially supported by German Bundusministerium für Furschung und Technologie under the grant No. 211-5291 YPI and by the grant INTAS-93-633.

\section{References}

[1] J.B. Kogut, Phys.Rev. D21 (1980) 2316.

[2] N.S. Ananikian and A.Z. Akheyan, Sov.J.Nucl.Phys. 53 (1991) 1143.

[3] A.Z. Akheyan and N.S. Ananikian, J.Phys.A:Math.Gen. 25 (1992) 3111.

[4] R.B. Griffiths, Phys.Rev.Lett. 21 (1970) 715; M. Blume, V.J. Emery and R.B. Griffiths, Phys.Rev. A4 (1971) 1071.

[5] J.W. Essam and M.E. Fisher, Rev.Mod.Phys. 42 (1970) 272.

[6] R.C. Edgar, Nucl.Phys. B200 (1982) 345.

[7] G. Bhanot, J.M. Drouffe, A. Schiller and I.O. Stamatescu, Phys.Lett. B125 (1983) 67. 
[8] L. Turban, J.Phys.A:Math.Gen. 17 (1984) 419.

[9] A.R. Avakian, N.S. Ananikian and N.Sh. Izmailian, Phys.Lett. A150 (1990) 163.

N.S. Ananikian, A.R. Avakian and N.Sh. Izmailian, Physica A172 (1991) 391.

[10] N.S. Ananikian, N.Sh. Izmailian, and R.R. Shcherbakov, JETP Lett. 59 (1994) 71. 


\section{Figure 1.}

Phase diagram of the $\mathrm{Z}(3)$ lattice gauge theory. The $\lambda$-line of the second-order phase transition (solid line) and the line of the first-order phase transition (dashed line) meet together at the tricritical point A. These lines separate the plane of the coupling constants $\left(\beta_{g}, \beta_{2 g}\right)$ onto two regions (ordered and disordered). 
This figure "fig1-1.png" is available in "png" format from: http://arxiv.org/ps/hep-lat/9408011v1 